\title{
3D printed mountain area with augmented reality for education purposes
}

\author{
Dobrin Petkov \\ University of Architecture, Civil Engineering and Geodesy, 1, Chr. Smirnenski Blvd, 1046 Sofia, Bulgaria \\ dob_petkov@abv.bg
}

Keywords: 3D printer, augmented reality, 3D model, mountain area, education

\begin{abstract}
:
3D printing technique is increasingly used in nowadays. In the modern life of "digitalization", the line between imagination and the real world does not exist, because of the augmented reality, which is the ideal way to visualize objects. The combination of these two technologies can be a learning resource for students and the implementation of digital technologies in school disciplines is a step towards for modernizing the educational system. This article represents the creation of prototype of a 3D printed mountain area, which could be a "marker" that activates augmented reality. The aim is to add digital information to the physical object, such as: elements of the content of topographic maps, orthophotos, etc., for easier assimilation of the teaching material in cartography and visual and physical representation of geography.
\end{abstract}

One of the main challenges for teachers is to capture the attention and stimulate the motivation and activity of students in the learning process. With each succeeding generation, the pupils and students change, which sets the requirement for a change in the methodology so that it is always appropriate and fascinating. Choosing the right learning technology is extremely important for the teacher planning digital learning. It must be at the same time accessible for use, pedagogically effective in view of the set methodological goals and tasks. Mountain maps in traditional paper form are very useful and will continue to be used for several years in the future. But the development of technologies such as multimedia and interactivity gives a chance to find a new cartographic representation of mountain areas, which will become a very useful product in the future. According to prof. Bandrova (2005), "3D provides opportunities for analyzing information that is not present in 2D presentation."

3D printing is a modern technology for making a 3D object of any shape, using a digital model. This is a cumulative process in which layer after layer accumulates to form different objects. 3D printing consists of three main steps modeling (creating a model using CAD software), printing (structuring the layers of liquid, dust particles, paper, etc.) and finishing (polishing and adding color) (Asenova A., Afzali J., 2018). Among the most commonly used materials in threedimensional printing are polylactic acid (PLA, a type of polyester that is biodegradable and bioactive when heated). Three-dimensional printing is used both in the production of prototypes and in many different areas. The scientists are still looking for new applications of this type of technology. During the introduction of 3D printing in the learning process, students go through several stages: analyzing the specific learning content, prototype planning, model construction, creating the prototype using a 3D printer and reflection. Within this process, learners achieve increasing control over information, process it, extract the essential, include it in the system of their available experience.

Augmented reality (AR) is a direct or indirect real-time view of a physical, real environment, the elements of which are "added" by computer-generated information. AR is used to improve the natural environment or situations. With the help of AR, the information about the surrounding real world of the user becomes interactive and digitally manipulable (Trajkovski K., 2018). Information about the environment and its objects are superimposed on the existing environment. This information can be virtual or real. AR can be based on an image or an object. The target image can be any raster image with enough distinguishing points. Teaching cartography and geography in an augmented reality environment amplifies the traditional means and it is carried out quickly, easily and interestingly. The motivation of students and pupils increases because it creates interest in the studied learning content. The training is based on the means of communication and information. They gain confidence and self-confidence that they create in a familiar environment - figuratively and sensually closer to them and create learning models using computer-generated images (Lateva R., 2018).

3D printing and augmented reality are examples of how technologies are extremely evolving. 3D printing and augmented reality are suitable for every user, regardless of their technological experience. In the future, a study with students will be carried out, presenting an experimental plan, showed in Figure 1, which will lead to the creation of a prototype for educational purposes. The 3D printed mountain area will be created, based on a digital terrain model, on a scale limited to the capabilities of the 3D printer. This printed model will be the "marker", which must be scanned using a special 
application to activate augmented reality. Various data, such as satellite images, horizontals, ridges, ravines, etc., can be used by superimposing on the 3D model.

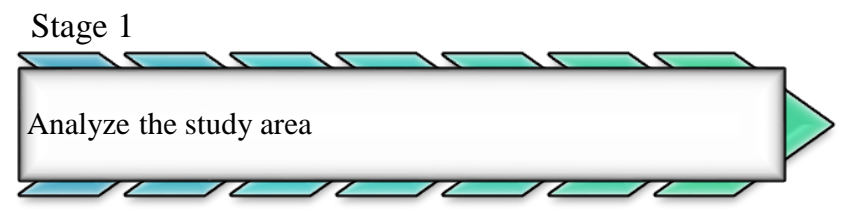

Stage 2
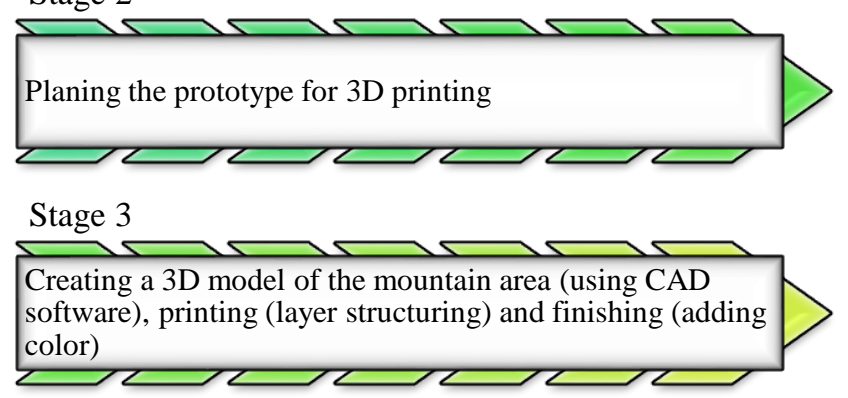

Stage 4

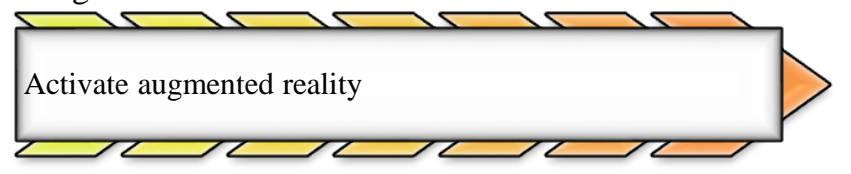

Figure 1. Experimental educational plan.

\section{Acknowledgements (optional)}

The author would like to thank prof. Temenoujka Bandrova for her comments and to collaborators from Bulgarian Cartographic Association who took part in this work.

\section{References (optional)}

Asenova A., Afzali J. (2018). Application of technology for 3D printing in education, New Bulgarian University

- Sofia, Bulgaria, Yearbook Telecommunications 2018, vol.5, ISSN２534-854X（online) http://www.telecommunications.nbu.bg/bg/godishnik (in Bulgarian).

Bandrova T., (2005). Innovative Technology for the Creation of 3D Maps, Data Science Journal, Vol.4, pp. 53-58.

Harding C., Hasuik F., Wood A., (2021). TouchTerrain - 3D Printable Terrain Models, Multidisciplinary Digital Publishing Institute, ISPRS Int. J. Geo-Inf. 2021, 10(3), 108; https://doi.org/10.3390/ijgi10030108.

Lara-Prieto V., Bravo-Quirino E., Rivera-Campa A., Gutiérrez-Arredondo J., (2015). An Innovative Self- learning Approach to 3D Printing Using Multimedia and Augmented Reality on Mobile Devices, 2015 International Conference on Virtual and Augmented Reality in Education.

Lateva R., (2018). Augmented reality and live streaming in classroom, Edition: no.2, 2018, Trakia University, Stara Zagora.

Trajkovski K. (2018). Augmented Reality Display of a Mountainous Region, 11th Mountain CartographyWorkshop, 2018, Hvar, Croatia.

Yonov N., (2019). School Atlas with Augmented Reality, Proceedings of the International Cartographic Association, 2 , 2019. 29th International Cartographic

Conference (ICC 2019), 15-20 July 2019, Tokyo, Japan. 